\title{
Is Immuno-modulation the Key to COVID-19 Pandemic?
}

\author{
Ajay Gupta ${ }^{1}$ \\ Received: 1 April 2020 / Accepted: 15 April 2020 / Published online: 27 April 2020 \\ (c) Indian Orthopaedics Association 2020
}

\section{Dear Editor,}

World, presently, is undergoing the worst ever health crisis with sudden influx of the dreaded virus COVID-19. This is the time when all medical specialities with all their curative skills and scientific knowledge should come forward and participate in the present war against COVID-19. The Orthopaedic fraternity has proved its innovative skills fighting diverse diseases from tuberculosis to gap non-unions, and can surely give a helping hand or vision to our fellow colleagues.

COVID-19 infection is the biggest threat today for survival of the human race. It has a faster spread and has increased mortality rate than the other influenza viruses. Individuals who are elderly or who have underlying chronic health problems are at a higher risk of mortality. A dysregulated and enhanced immune response called cytokine storm than the virus itself is described to produce respiratory failure, acute respiratory distress syndrome (ARDS) and multiple organ dysfunction syndrome (MODS) leading to death in these patients [1].

Human body has two types of immunity called type 1 or type 2 immunity invoked through Th1 cells and Th2 cells to rationalize virtually all the known patterns of immune response. Th1 cells are known to be induced by intracellular pathogens like Mycobacteria, Salmonella, leishmaniasis, viruses, etc. These are involved in evoking the classic delayed-type hypersensitivity (DTH) skin response to viral and bacterial antigens, and fight cancer cells. Th2 cells are believed to emphasize protection against extracellular pathogens such as multicellular parasites. The Th1 pathway is often portrayed as being the more aggressive of the two and release various proinflammatory cytokines like IL1, IL2, IL12, TNF- $\alpha$, IFN- $\gamma$, etc. while the Th2 pathway is seen to

\footnotetext{
Ajay Gupta

drajaygupta@hotmail.com

1 Department of Orthopaedics, Maulana Azad Medical College and Associated LN Hospital, New Delhi 110002, India
}

release various anti-inflammatory cytokines like IL4, IL5, IL6, IL10, etc. which are also involved in several allergenspecific and related IgE-based events like development of airway eosinophilia, the induction of airway smooth muscle contraction, mucus production, etc. and also predisposing to several systemic autoimmune diseases [2-5]. Factors like malnutrition, overcrowding, vitamin D deficiency, etc. are also known to induce Th2 immunity which in turn downregulates the available Th1 immunity [6-9].

The vast majority of elderly population in developed nations like Italy or USA, due to their less exposure to various infective antigens, have reduced Th1 immunity [10]. The stock of their naïve $\mathrm{T}$ cells too remains in the age-related relatively dysfunctional state. It is important to note that COVID-19 has inflicted high morbidity and mortality to only that segment of the world population, [11] which has low Th1 immunity due to poor or no exposure to strong Th1 antigens like Mycobacteria, Salmonella, even Bee stings, etc.

COVID-19 has been seen to affect more of those countries who have robust and free influenza vaccination program leading to reduced natural flu infection among their population [12-14]. Immunity from disease often follows a single natural exposure to it, while immunity from vaccines usually develops after several doses only. The natural flu infection is known to provide better immunity and protection with higher antibody (IgA) level than after the flu vaccination. This is attributed to the different route of antigen presentation. Intramuscular route of vaccination fails to induce antibodies which can only be produced after mucosal viral replication in mucosal cells of the respiratory tract in natural infection [15]. Another possible reason for this viral outburst could be adaption to an unnatural way of life, depriving it of such a common and natural Th1 immunity of common flu.

Influenza virus is uniquely characterized by frequent mutations due to antigenic drifts and antigenic shifts. To ensure optimal vaccine efficacy against prevailing strains, the antigenic composition of the vaccine is revised twice annually in both the northern hemisphere (NH) and southern hemisphere $(\mathrm{SH})$, and contemplates likely antigenic 
characteristics of the continuously changing future mutations of viruses within the WHO Global Influenza Surveillance and Response System (GISRS). WHO recommends influenza vaccine with different strains for the $\mathrm{NH}$ and $\mathrm{SH}$. Is it not strange that influenza vaccine, meant for those living in northern hemisphere, is also available and being used in India, while WHO recommendations on vaccine formulation for India strongly favour the Southern hemisphere vaccine rather than the one for Northern hemisphere? [16]. Immunization rate against influenza is significantly higher in highincome (92\%) than in low-income countries (8-47\%) [12]. Higher cost of the influenza vaccine, its adverse effects and rapid change in nature and epidemiology of the virus are reported to be the cause for its poor coverage among the south Asian population [17]. The poor flu vaccination coverage among our population too has unknowingly helped the society to maintain its natural immunity and fight better with this global enemy COVID-19.

Countries like Italy, Spain, Germany etc. with very low prevalence of tuberculosis and where the routine $\mathrm{BCG}$ immunization has been discontinued since decades, [18-20] are witnessing the worst form of this global epidemic of Corona virus [11]. A relatively elderly population of these countries with many co-morbid conditions is slated to be the possible explanation for such an occurrence but their low Th1 immunity due to the stray past exposure to various infectious antigens, particularly the Mycobacteria, cannot be just ignored which could be further substantiated with the relatively low COVID-19 deaths in countries like India, Indonesia, Vietnam and Nigeria [11].

BCG is the live attenuated vaccine used against tuberculosis prepared from M. bovis bacillus. It is one of the most widely used vaccines and is part of national vaccine program of more than 154 countries. BCG is estimated to be given to more than 100 million children per year globally and, thus, has a well-established safety profile. BCG, besides providing specific protection against tuberculosis, has the capacity to confer relatively broad-spectrum protection against other pathogens also like candida, staphylococcus, etc. [21]. BCG provides this broad spectrum or the cross protection by making certain changes in specific genes of circulating monocytes or the natural killer (NK) cells [22,23]. These changes can be considered memory like responses in innate immunity cells, which, otherwise, are part of adaptive immunity only. BCG-vaccinated children have not only shown decreased mortality rate associated with malaria, diarrhea, attenuated yellow fever virus, etc. [24], but also have shown reduced risk of developing acute lower respiratory tract infections also in separate studies [25, 26]. BCG vaccination has also shown reduced respiratory infection in many RCTs in adults and in the elderly population as well $[27,28]$. Protective effect of BCG wanes with time and is reported to disappear completely in $10-15$ years
[29]. Countries with policy of compulsory BCG vaccination at birth, therefore, may provide protection to their population from childhood tuberculosis but the continued collateral benefits like what is being contemplated during the present COVID-19 pandemic are possible only through the environmental exposure to Mycobacteria as seen in the tubercular high burden countries.

It has been shown that influenza provides significant protection against the Mycobacterial infection [30]. It is possible that a reciprocal cross immunity exists between the two pathogens and will be the most deserving need of the hour at a time when the whole world is struggling to fight with the Corona outbreak. Any modality of treatment or prophylaxis of even a meagre hope would be worth a trial of its clinical application. Mice coinfected with BCG and influenza A virus exhibited reduced frequency and numbers of CD8 $\mathrm{T}$ cells specific to Mycobacterium (BCG) in the lungs when compared with mice infected with BCG alone. Viral-specific CD8 T cells were seen to have special affinity towards BCGinduced granulomas resulting in substantial accumulation of viral-specific CD8 T cells there [31]. Such an affinity can be made to be used in patients with COVID-19 to divert and subvert its overactive immune response towards BCG granulomas. It may be inferred that deployment of BCG-specific CD8 T cells will occur at the cost of viral-specific CD8 T cells, thus reducing the magnitude of cytokine storm of the Corona infection.

Acute viral infections like influenza or COVID-19 normally result in the formation of short-lived, but highly functional, terminally differentiated effector Th1 cells that eliminate infected target cells [32]. Influenza virus in an experimental study on mice has shown to be lethal only if it is able to induce a combined Th1 and Th2 response [33]. Similarly, cytokine storm with very high levels of diverse cytokines profile with mixed Th1/Th2 affinity like IL2, IL7, IL10, GCSF, IP10, MCP1, MIP1A, and TNF $\alpha$ were seen in ICU patients than non-ICU patients of COVID-19 infection. What prompts this unusual Th2 response in these patients could be the key to understand and evolve protective measure against this huge threat to humanity [34]. Another study comparing immunological profile of mild or severe COVID-19 patients, low levels of CD4 $+\mathrm{T}$ and CD8 $+\mathrm{T}$ and very high levels of IL- 6 and IL-10 were reported in severe COVID-19 patients [35]. Mycobacteria is one of the strongest antigens to induce Th1 immunity [36]; while, Salmonella has also been considered a model pathogen for inducing strong Th1 response [37]. Vaccination of both of these pathogens shall restore the forgotten and decreased Th1 immunity among the immunologically useful antigen starved population of the developed nations, shall downregulate the Th2 bias environment of cytokine storm with IL6 and, thus, help reducing local tissue destruction in COVID19 patients [38]. 
BCG-specific $\mathrm{CD}^{+}{ }^{+} \mathrm{T}$-cell response is reported to be peaked 6-10 weeks after vaccination [39]. BCGs prophylactic use, therefore, may not yield instant protection as desirable, yet is a tool with little possibility to lose but enormous possibility of gains. Under the present circumstances, when developing a new specific vaccine against COVID-19 may take years to reach the target population, BCG can prove to be a good stop gap measure and may actually prove miraculous. Considering its wide and easy availability, considering its low cost, excellent safety profile and considerable nonspecific immunogenicity including possible cross immunity against influenza, BCG vaccine should be considered for its prophylactic usage against COVID-19 in high-risk health professionals and the elderly population besides the use of chloroquine [40]. It is suggested that a study of critically ill patients of COVID-19 be undertaken in high prevalence tuberculosis countries like ours to measure their level of immunity against Mycobacteria through Mantoux tuberculin skin test or interferon-gamma release assays (IGRAs). A relatively low-test reading among these patients than those of the general population shall support the hypothesis that population with low or no immunity against Mycobacteria are more prone to COVID-19 infection and shall possibly benefit with $B C G$ vaccine.

\section{Compliance with Ethical Standards}

Conflict of interest The authors declare that they have no conflict of interest.

Ethical standard statement This article does not contain any studies with human or animal subjects performed by the any of the authors.

Informed consent For this type of study informed consent is not required.

\section{References}

1. Mehta, P., Mc Auley, D. F., Brown, M., et al. (2020). Covid-19, consider cytokine storm syndromes and immunosuppression. Lancet. https://doi.org/10.1016/S0140-6736(20)30628-0.

2. Roitt, I., Brostoff, J., \& Male, D. (1998). Immunology (Fifth Edition). Philadelphia: Mosby.

3. Wang, L., Cai, Y., Cheng, Q., Hu, Y., \& Xiao, H. (2002). Imbalance of Th1/Th2 cytokines in patients with pulmonary tuberculosis. Zhonghua Jie He He Hu Xi Za Zhi, 25(9), 535-537.

4. Collins, F. M. (1979). Cellular antimicrobial immunity. Critical Reviews in Microbiology, 7, 27-91.

5. Bretscher, P. A. (1992). An hypothesis to explain why cell-mediated immunity alone can contain infections by certain intracellular parasites and how immune class regulation of the response can be subverted. Immunology and Cell Biology, 70, 343-351.

6. Strachan, D. P. (1997). Allergy and family size: a riddle worth solving. Clinical and Experimental Allergy, 27, 235-236.
7. González-Torres, C., González-Martínez, H., Miliar, A., et al. (2013). Effect of malnutrition on the expression of cytokines involved in Th1 cell differentiation. Nutrients., 5, 579-593.

8. Neyestani, T. R., Woodward, W. D., \& Hillyer, L. (2004). Serum levels of Th2-type immunoglobulins are increased in weanling mice subjected to acute wasting protein-energy malnutrition. Iranian Journal of Allergy, Asthma and Immunology, 3, 1-6.

9. Salahuddin, N., Ali, F., Hasan, Z., Rao, N., Aqeel, M., \& Mahmood, F. (2013). Vitamin D accelerates clinical recovery from tuberculosis: results of the SUCCINCT Study (Supplementary Cholecalciferol in recovery from tuberculosis). A randomized, placebo-controlled, clinical trial of vitamin D supplementation in patients with pulmonary tuberculosis'. BMC Infectious Diseases, 19, 22. https://doi.org/10.1186/14712334-13-22.

10. Okada, H., Feillet, H., \& Bach, J.-F. (2010). The "hygiene hypothesis" for autoimmune and allergic diseases: an update. Clinical and Experimental Immunology, 160(1), 1-9.

11. Covid-19 coronavirus pandemic. Available and collected on 30th March 2020 from https://www.worldometers.info/coronavirus

12. Principi, N., Camilloni, B., \& Esposito, S. (2018). Influenza immunization policies: Which could be the main reasons for differences among countries? Human Vaccines and Immunotherapeutics, 14(3), 684-692.

13. Di Pietro, A., Visalli, G., Antonuccio, G. M., \& Facciola, A. (2019). Today's vaccination policies in Italy: The national plan for vaccine prevention 2017-2019 and the Law 119.2017 on the mandatory vaccinations. Annali di Igiene, 31(2 suppl 1), 54-64.

14. Panatto, D., Lai, P. L., \& Mosca, S. (2020). Influenza vaccination in Italian healthcare workers (2018-2019 Season): strengths and weaknesses. Results of a cohort study in two large Italian hospitals. Vaccines, 8, 119.

15. Chan, K.-H., To Kelvin, K. W., Hung Ivan, F. N., et al. (2011). Differences in antibody responses of individuals with natural infection and those vaccinated against Pandemic H1N1 2009 influenza. Clinical and Vaccine Immunology, 18(5), 867-873.

16. Thakre, R., \& Patil, P. S. (2019). Influenza vaccine paradox. Indian Paediatrics, 56, 80.

17. Wiwanitkit, V. (2019). Influenza vaccination: some clinical concerns for South Asian practitioner. Ind J Med Res., 150(2), 107-109.

18. Zwerling et al (2011) The BCG world atlas: a database of global vaccination policies and practices. PLos Medicine 8(3)

19. Faust, L., Schreiber, Y., \& Bocking, N. (2019). A systemic review of BCG vaccination policies among high risk groups in low TBburden countries. BMC Public Health, 19, 1504.

20. Global tuberculosis report (2019) WHO's global tuberculosis database. who.int/tb/country/data

21. Covian, C., Fernandez-Fierro, A., Retamal-Díaz, A., et al. (2019). BCG-induced cross-protection and development of trained immunity: implication for vaccine design. Frontiers in Immunology, 29, 2806.

22. Netea, M. G., \& van Crevel, R. (2014). BCG-induced protection: effects on innate immune memory. Seminars in Immunology, 26(6), 512-517.

23. Arts, R. J. W., Carvalho, A., La Rocca, C., Palma, C., Rodrigues, F., Silvestre, R., et al. (2016). Immunometabolic pathways in BCG-induced trained immunity. Cell Reports, 17, 2562-2571.

24. Roth, A., Gustafson, P., Nhaga, A., Djana, Q., Poulsen, A., Garly, M. L., et al. (2005). BCG vaccination scar associated with better childhood survival in Guinea-Bissau. International Journal of Epidemiology, 34(3), 540-547.

25. Hollm-Delgado, M. G., Stuart, E. A., \& Black, R. E. (2014). Acute lower respiratory infection among Bacille Calmette- Guérin (BCG)-vaccinated children. Pediatrics, 133(1), e73-81.

26. de Castro, M. J., Pardo-Seco, J., \& Martinón-Torres, F. (2015). Nonspecific (heterologous) protection of neonatal BCG 
vaccination against hospitalization due to respiratory infection and sepsis. Clinical Infectious Diseases, 60(11), 1611-1619.

27. Wardhana, E. A., Datau, E. A., Sultana, A., et al. (2011). The efficacy of Bacillus Calmette-Guerin vaccinations for the prevention of acute upper respiratory tract infection in the elderly. Acta Medica Indonesiana, 43, 185e90.

28. Ohrui, T., Nakayama, K., Fukushima, T., et al. (2005). Prevention of elderly pneumonia by pneumococcal, influenza and BCG vaccinations. Nihon Ronen Igakkai Zasshi, 42, 34e6.

29. Whittaker, E., Nicol, M. P., Zar, H. J., Tena-Coki, N. G., \& Kampmann, B. (2015). Age-related waning of immune responses to BCG in healthy children supports the need for a booster dose of BCG in TB endemic countries. Scientific Reports., 8, 15309.

30. Gupta, A., Gupta, A., Kumar, A., \& Arora, S. (2016). Immunotherapy for non-responders among patients of spinal tuberculosis. Indian Journal of Tuberculosis, 63(2), 79-85.

31. Florido, M., Grima, M. A., Gillis, C. M., et al. (2013). Influenza A virus infection impairs Mycobacteria-specific T cell responses and Mycobacterial clearance in the lung during pulmonary coinfection. Journal of Immunology, 2013, 191.

32. Kahan, S. M. (2015). T cell exhaustion during persistent viral infections. Virology, 479-480, 180-193.

33. Turianova, L., Lachova, V., Svetlikova, D., et al. (2019). Comparison of cytokine pro les induced by nonlethal and lethal doses of influenza A virus in mice. Experimental and Therapeutic Medicine, 18, 4397-4405.

34. Huang, C., Wang, Y., Li, X., et al. (2020). Clinical features of patients infected with 2019 novel coronavirus in Wuhan China. Lancet, 395(10223), 497-506.
35. Wan S, Qingjie YE and Fan S, et al (2020) Characteristics of lymphocyte subsets and cytokines in peripheral blood of 123 hospitalized patients with 2019 novel coronavirus pneumonia (NCP) MedRxiv. https://doi.org/10.1101/2020.02.10.20021832.

36. Diehl, S., \& Rincon, M. (2002). The two faces of IL-6 on Th1/ Th2 differentiation. Molecular Immunology, 39, 531-536.

37. Lyadova, I., \& Panteleev, A. V. (2015). Th1 and Th17 cells in tuberculosis: protection, pathology, and biomarkers. Mediators of Inflammation, 2015, 854507.

38. O'Donnell, H., \& MsSorley, S. (2014). Salmonella as a model for Non-Cognate Th1 cell stimulation. Front Immunology, 5, 621.

39. Soares, A. P., Kwong Chung, C., Choice, T., et al. (2013). Longitudinal changes in CD4+ T-cell memory responses induced by BCG vaccination of newborns. Journal of Infectious Diseases, 207(7), 1084-1094.

40. Wang, M., Cao, R., Zhang, L., et al. (2020). Remdesivir and chloroquine effectively inhibit the recently emerged novel coronavirus (2019-nCoV) in vitro. Cell Research, 30, 269-271.

Publisher's Note Springer Nature remains neutral with regard to jurisdictional claims in published maps and institutional affiliations. 